\title{
A Power Control Based Cross-layer Communication Protocol for Wireless Sensor networks
}

\author{
Jiang Jingqi, Ji Peng, Sun Qiushuo, Zhang Songyang \\ School of Information Science and Engineering, Northeastern University, China \\ jiangjingqicansy@sohu.com, jipeng@ise.neu.edu.cn
}

\begin{abstract}
An effective communication protocol for wireless sensor networks should provide a good quality of service (QoS) for network data transfer, but the traditional layering protocol stack can't sufficiently meet this complex requirement. So a new cross-layer communication protocol - directed spanning tree-based power control (PCDST) is proposed. In the algorithm, a directed spanning tree routing algorithm is discussed to resolve unnecessary energy consumption during data transmission. Regarded as a key factor, transmission power of node should be controlled to decrease the intensity of communication contention. A NUM model is constructed to control power. Based on Prim-dual Algorithm, a Lagrange dual function is designed and optimal solution set of transmission power is deduced. Make use of these cross-layer optimal schemes, WSNs achieve energy efficiency and smooth communication mode. The simulation results show that the performance of the proposed protocol is much better in energy efficiency and improvement of communication throughput.

Index Terms - Wireless sensor networks; communication protocol; cross-layer optimization; power control; spanning tree.
\end{abstract}

\section{Introduction}

Wireless sensor networks (WSNs) are representative noninfrastructure networks which are capable of wireless communication [1]. WSNs have a large number of sensor nodes with micro-processing capability and limited power. These tiny nodes work in the order of a few to tens of hundreds in areas ranging from sophisticated urban homes to extremely hostile, remote areas. Different from the traditional networks, WSNs are data-centric, self-configuring and selfhealing, so it's deemed to be the most appropriate data processing method of Ambient Intelligence [2]. For the advanced technology and broad space of application, nowadays, WSNs have attracted plenty of research attention from the academia and industry. Since it's capable of attaining unaffected intercourse between human and computer, it is said that WSNs are the key of Artificial Intelligence [3].

Communication is one of the most important functions of WSNs, it's responsible for relaying sensed information of original nodes to sink node. Since the power of nodes and communication capacity of network are limited, there are some factors essential to communication, such as energy efficiency and traffic of transmission. The traditional protocol of ad hoc can not directly meet the needs of WSNs; and existing WSNs layered protocol stack also can't optimize the communication QoS totally. However, the cross-layer conception can balance the resource among physical layer, MAC layer and routing layer, and achieve the overall optimization of WSNs. So the studies on cross-layer optimization become the noble hot area of WSNs [4]. 1999, S.H. Low et al. [5] proposed a network flow control algorithm that is based on the construction of distributed optimization function. 2004, M. Zawodniok et al. designed a distributed power control based MAC protocol for communication resource allocation uncertainty [6]. 2005, M. Chiang et al. [7] discussed how to take power control of physical layer and congestion control of transport layer, which can improve network throughput and energy efficiency. Moreover, they [8] also detailedly discussed the decomposition mathematical issues of cross-layer optimization for wireless networks.

In order to take WSNs to have a good performance, a cross-layer communication protocol that named as directed spanning tree-based power control (PCDST) is proposed. In this protocol, the bottom three layers of the traditional networking stack-physical layer, MAC layer and routing layer are modeled together. In this way, a tree based communication protocol can effectively balance the energy consumption and reliability of the network, and it can meet the requirements of the high robustness of the Wireless sensor networks.

\section{Directed Spanning Tree Model Based on Geographic Location Information}

Spanning Tree is a typical multi-hop communication protocol of WSNs [9], which aims to connect all nodes in network as a communication tree which leads to aggregation node. All of the communication routings based on query and data communication needs of aggregation nodes. The advantages of this model are simple structure, distributed features, suitable for network self-configuring restructuring and data fusion. However, Spanning Tree has relatively loose structure, the network energy consumption and connectivity is more focused on local solutions, so it's difficult to balance the energy of the entire network.

Specific to the disadvantage of the spanning tree algorithm, a location-based information directed spanning tree model is proposed. To some extent, it can establish the contact between a single node and network global, reduce network energy loss on the whole, and improve the reliability of the communication.

\footnotetext{
* This work is partially supported by CNSF Grant \# 61203216 to Ji Peng and CNSF Grant \# 61273078 to Wu Chengdong.
} 
Different from normal Spanning Tree, Directed spanning tree model considers about how to save the total energy consumption of network. Therefore, each node should be directional control so that packets are sent more tend to sink node when selecting father node. Since the data transmission can be considered as efficient in data-centric WSNs when they are received by sink, the means of communication oriented to sink can avoid the invalid energy loss of in the other direction and reduce the time delay. So the Model should be established based on the following two assumptions: (1) in the network initialization stages, each node can get its own location and sink's location; (2)in the network initialization stages, all nodes have enough energy to send and receive data packets, and can transmit data to sink with multi-hop form.

Establishing a undirected weight graph $\mathrm{G}=(\mathrm{V}, \mathrm{E}, \mathrm{W})$ based on topology to represent wireless sensor networks. Where $V=\left\{v_{1}, \ldots, v_{\mathrm{n}}\right\}$ is a collection of nodes, Respectively corresponding to each wireless sensor nodes. $\mathrm{E}=\left\{e_{i j} \mid \mathrm{d}(i, j) \leqslant\right.$ $\left.R_{\text {imax }}\right\}$ is the set of edges, it means the undirected connection between any two nodes. However, $\mathrm{d}(i, j)$ is the Euclidean distance between node $v_{\mathrm{i}}$ and $\mathrm{v}_{\mathrm{j}} ; R_{\mathrm{imax}}$ is the maximum communication radius of node $v_{\mathrm{i}}$, which is proportional to the transmission power of $i$. When $d(i, j) \leqslant R_{\text {inax }}$ indicate that node is normal to transmit data to each other between $v_{\mathrm{j}}$ and $v_{\mathrm{i}} . W=\{\mathrm{w}(i, j)\}$ is the set of each side weight in network diagram, and $w(i, j)$ is the weights of the communication between node $v_{\mathrm{i}}$ and $v_{\mathrm{j}}$. By defining $w(i, j)$, reasonable neighbor nodes can be choose for nodes as data relay node in order to establish a reliable routing. There are a number of elements to determine the weight value, to build a directional routing protocol. $w(i, j)$ can be defined as formula (1).

$$
w(i, j)=\frac{d(i, S)-d(j, S)}{P_{i}}
$$

Where $d(i, S)$ and $d(j, S)$ are nodes $v_{\mathrm{i}}$ and $\mathrm{v}_{\mathrm{j}}$ Euclidean distance to the sink node Sink, their value can be obtained through the node position information; $P_{\mathrm{i}}$ is the data transmission power of the node $v_{\mathrm{i}}$. According to the Formula (1), weights $w(i, j)$ just be related to $d(i, S)-d(j, S)$ and the power $P_{\mathrm{i}}$ of source node $v_{\mathrm{i}}$. As shown in Fig. 1 , and the value of $d(i, S)-d(j, S)$ determines the data transmission directivity in essence. When the value of $d(i, S)-d(j, S)$ is positive and large, the corresponding neighbor node $v_{\mathrm{j}}$ close to the sink node in the direction, invalid energy costs and the communication delay of node vi sending data is little. Therefore, when the power $P_{\mathrm{i}}$ of node $v_{\mathrm{i}}$ data transmission is sure, node $v_{\mathrm{i}}$ can completely choose the father node as communication objectives based on the value of weight $\mathrm{w}(i, j)$, i.e. $d(i, S)-d(j$, $S$ ). The entire wireless sensor networks can also based on the principle of the father node selection of node to establish directed spanning tree routing protocol based on geographic location information. To implement the algorithm, it is important to choose the optimal transmission of any node $v_{\mathrm{i}}$ in WSNs. Therefore it is necessary to establish cross-layer desired node for transferring power control model according to the network global optimization, and solve the optimal node transmission power solution set $P_{\text {opt }}=\left\{P_{\mathrm{i} o p t} \mid i \in n\right\}$ 。

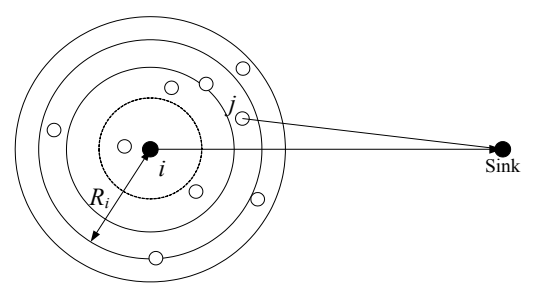

Fig.1 The mechanism of father node selection in directed spanning tree

\section{The Establishment and Solution of Power Control model Network-oriented Global Optimization}

\section{A. The establishment of network optimization model based on power control}

The data transmission power of node is an important parameter of WSNs, it directly affect the QoS of network. If the transmission power is too low, part of the node can not establish communication links, resulting in fragmentation of the network; If the transmission power is too high, though the connectivity of the network is ensured, too many nodes involved in the communication increase the competitive strength of the network, resulting excessive consumption of energy, communication data is easy to packet loss or misinformation, resulting in decreased overall network performance. For the spanning tree algorithm, the intensity of competition is more important. This is because the network inner layer node in communication tree routing (close to Sink in position) have a greater opportunity to become the father node of some of the nodes, accept more onerous task communication, and consume more energy. Therefore, it become the key to improve the performance of the spanning tree routing algorithm that how to optimize the size of the transmission power of each node in WSNs to reasonable control the number of neighbor nodes which communicate with relaying node (father node) in communication, and effectively reduce network competition intensity and energy consumption.

Based on the above ideas, it can be set that the maximum communication competitive strength of each in sensor networks is $N_{\mathrm{j}}$, the values of $N_{\mathrm{j}}$ is related to node location, the closer to Sink the greater the value. So that the maximum communication nodes set of all nodes in WSNs $N=\left\{N_{\mathrm{j}} j \in n\right\}$ can be obtained. At the same time, Parameter matrix $K=\left(k_{j i}\right)$ is established in which $k_{j i}$ is the communication cost parameters between $v_{\mathrm{i}}$ and $v_{\mathrm{j}}$, it is determined by the number of hops between sending node and aggregation node, which characterize the possibility of communication about $v_{\mathrm{i}}$ and $v_{\mathrm{j}}$. According to the entire network connectivity, it can be stipulate that $k_{j i}$ meet the following conditions:

$$
k_{j i}= \begin{cases}k_{p} & d(i, j) \leq R_{i \max } \text { and } i \neq j \\ 0 & d(i, j) \geq R_{i \max } \\ 0 & i=j\end{cases}
$$


According to the number of hops between sending node and aggregation node, the value of $k_{p}$ is in $[0,1]$, and $k_{p} P_{\mathrm{i}}$ can reflect the competitive strength of a sending node to communicate its neighbor nodes. For each father node in spanning tree, the whole competitive strength it endured from child nodes must be less than the set maximum communication intensity of competition. Based on the the thought of network utility maximization (Network Utility Maximum, NUM ), setting a utility function $U_{T}\left(P_{\mathrm{i}}\right)$, which can be measured and associated with the data transmission power for each node. Set the function monotonically increasing and be concave, network global optimization constraint model can be built as followed.

$$
\begin{array}{cc}
\max & \sum_{i=1}^{n} U_{T}\left(P_{i}\right) \\
\text { s. t. } & K P \leq N \\
& P_{i} \geq 0
\end{array}
$$

Through solving the constraint model, the optimal transmission power satisfies the demand of global technology of wireless sensor networks can be obtained to control the transmission power of the network.

\section{B. Solving the optimization based on Lagrange Duality}

To meet the overall WSNs technology needs, optimization problem in (3) should be solved based on duality theorem. First, the Lagrange Duality equation of optimization problem in (3) is as followed:

$$
\begin{aligned}
D(\lambda) & =\max _{P_{i} \geq 0}\left\{\sum_{i=1}^{n} U_{T}\left(P_{i}\right)-\lambda^{k}(K P-N)\right\} \\
& =\max _{P_{i} \geq 0}\left\{\sum_{i=1}^{n} U_{T}\left(P_{i}\right)-P_{i} \sum_{j=1}^{n} k_{j i} \lambda_{j}\right\}+\lambda^{k} N
\end{aligned}
$$

Where $\lambda=\left(\lambda_{j}\right)$ is Lagrange multiplier vector, and the physical meaning of $\sum_{j=1}^{n} k_{j i} \lambda_{j}$ is energy node $v_{\mathrm{j}}$ required as relaying node in data transmission. Based on the power consumption results $\sum_{j=1}^{n} k_{j i} \lambda_{j}$ of node $v_{\mathrm{i}}$, system can effectively assess the power $P_{\mathrm{i}}$ of their possible child nodes $v_{\mathrm{i}}$, and deduce the solving equation of $P_{\mathrm{i}}$ according to (4), such as shown in equation (5).

$$
P_{i}=\arg \max _{P_{i} \geq 0}\left\{\sum_{i=1}^{n} U_{T}\left(P_{i}\right)-P_{i} \sum_{j=1}^{n} k_{j i} \lambda_{j}\right\}
$$

According to equation (4), dual model of the original problem in (3) can be easy to solved as followed:

$$
\begin{array}{ll}
\min & D(\lambda) \\
\text { s.t. } & \lambda_{j} \geq 0
\end{array}
$$

System can optimally solve the model in equation (6) with the projected gradient method, the formula is as followed:

$$
\lambda_{j}(t+1)=\left[\lambda_{j}(t)-\rho_{j}\left(N_{j}-\sum_{j=1}^{n} k_{j i} P_{i}\right)\right]^{+}
$$

Where $\rho_{j}$ is the gradient solution step that is determined by system parameters, and []$^{+}$shows the solution process of the formula is project in the set of positive real numbers $R^{+}$, which shows primal-dual models and its solving relationship. Formula (5) and (7) can be iterative solving according to primal-dual algorithm, node data transmission power $P_{\mathrm{i}}$ which can achieve network utility maximization can be obtained.

In wireless sensor networks, after determining the optimal transmission power of the node itself, weights of the side communicate with the neighbor node can be obtained according to the weight formula in (1), and the neighbor nodes of the maximum weight side can be selected as communication father node in order to build the entire network to a entire communication spanning tree.

\section{Simulation Experiments and Results Analysis}

The purpose of simulation experiments is to verify that PCDST is effective in ensuring reliable communication of wireless sensor networks and improving the quality of communication services. The wireless sensor networks system structure of the simulation is as followed: $10-240$ sensor nodes are random deployed in the square area $100 \mathrm{~m} \times 100 \mathrm{~m}$ and be stationary after network topology is generated. Sink node at coordinates $(50,50)$, the energy of it is unrestricted, and the initial energy of other nodes is $0.5 \mathrm{~J}$, node runs out of energy means the node dead. The data packets of node send and receive is $1.2 \mathrm{Kbps}$, the communication distance node maximum transmission power can reach is $20 \mathrm{~m}$. A wireless sensor networks model of 100 nodes The simulation establish to study the status of the data transmission of nodes in PCDST protocol, and emulate based on simulating the status of routing connectivity in PCDST.

The simulation experiment also compare the performance of the algorithm and the correlation algorithm of the algorithm in this paper. There are two algorithms be compared, one of these is the Minimum Energy Spanning Tree (MEST) improved designed based on traditional spanning tree, another is typical geographic routing protocol-Geographic Random Forwarding (GeRaF). Experiment mainly simulate the two performance indicator of network quality of service, one of which is the condition of network lifetime change with the number of nodes - purpose to study the condition of protocol influencing network energy consumption; another is the situation of the number of the data packet aggregation node receives change with the cycle data packet generate-purpose to reflect the performance security of different protocols to network information throughput. Fig. 2 and Fig. 3 are the comparison of simulating these two performance parameters. 


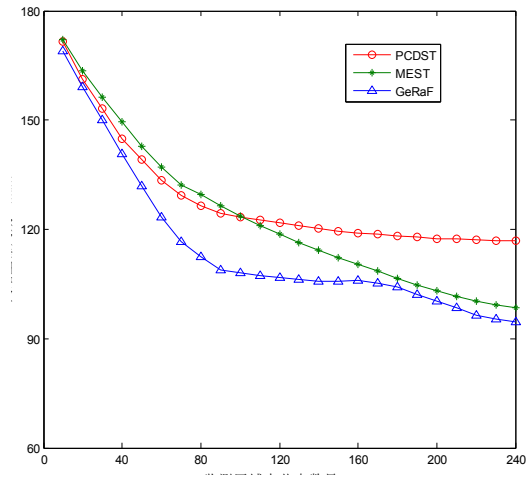

Fig.2 Network lifetime performance comparison among PCDST and other considered algorithms

It can be known through comparing and analysis that PCDST has good performance in extending network lifetime and improving the throughput of network communication. When the number of nodes reduces, the network lifetime is bound to reduce. But comparing to MEST and GeRaF, PCDST can effectively extend the network lifetime which make the network work better. This good performance thanks to the orientation strategy and power control algorithm PCDST adopt, which can properly schedule the communication energy and reduce unnecessary energy consumption. Though the relatively complex algorithms will generate a certain amount of reaction in energy savings when the number of nodes is relatively small, it is the reason why the efficacy of PCDST is lower than MEST when nodes are few; but as the number of nodes increasing, the energy-saving features of PCDST increasingly prominent.

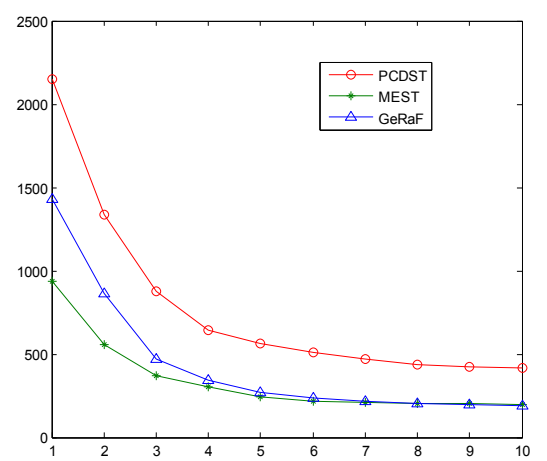

Fig.3 Received packets comparison among PCDST and other considered algorithms when $\mathrm{N}=200$ nodes

For data-centric wireless sensor networks, the network throughput characterized by the number of packets received by the sink node per unit time represents the work status of the network, if the throughput is too low, network communication will bottleneck and negative impact the transmission efficiency of the entire network. Comparing to MEST and GeRaF, PCDST will effectively protect the number of data packet sink node receives.

Therefore the wireless sensor networks applying PCDST has greater network throughput, which also means that it works and communicates in good condition. All these prove the power control algorithm of PCDST and the network global optimization mechanism, which effectively reduce the intensity of network traffic competition and avoid relatively concentrate plan of father node, in order to ensure the reliability of the network communication.

\section{Conclusion}

For the requirements of QoS in wireless sensor networks, a cross-layer routing protocol algorithm-power control based directed spanning tree (PCDST) is proposed in this paper. The algorithm defines a new weight formula based on the node location information and establishes the directed father node selection model to reduce the invalid energy consumption of communication. In order to reduce the competition of communication and improve WSNs throughput, the protocol establishes a power control model that can achieve network global optimization. Based on the original-dual algorithm, the model is optimized solved to schedule optimal data transmission power for each node. The results of simulation experiment show that PCDST can reduce the overall energy consumption of the network and extend the network lifetime as well as effectively improve network communication throughput and make WSNs work efficient and stable.

\section{Acknowledgment}

The authors thank National Nature Science Foundation for the NSF funds [The number of fund is No. 61203216 and No. 61273078].

\section{References}

[1] J. Gehrke, Ling Liu, "Guest editors' introduction: sensor-network applications," IEEE Internet Computing, vol. 10, no. 2, pp. 16-17, February 2006.

[2] Y. Zou, K. Chakrabarty, "Distributed coverage and connectivity centric technique for selecting active nodes in wireless sensor networks," IEEE Transactions on Computers, vol. 54, no. 1, pp. 978-991, March 2005.

[3] Z. Zhou, S. Zhou, S. Cui, "Energy-efficient cooperative communication in a clustered wireless sensor network," IEEE Transaction on Vehicular Technology, vol. 57, no. 6, pp. 3618-3628, 2008.

[4] I. Marin, J. Arias, E. Arceredillo, "LL-MAC: a low latency MAC protocol for wireless self-organised networks," Microprocessors and Microsystems, vol. 23, no. 11, pp. 1722-1734, 2007.

[5] W. Ye, J. Heidemann, D. Estrin, "An energy-efficient MAC protocol for wireless sensor networks," Proceedings of the 21st International Annual Joint Conference of the IEEE Computer and Communications Societies, June 2002, pp. 1567-1576.

[6] W. Ye, J. Heidemann, D. Estrin, "Medium access control with coordinated, adaptive sleeping for wireless sensor networks," IEEE/ ACM Transactions on Networking, vol. 12, no. 3, pp. 493-506, June 2004.

[7] V. Raisinghani, S. Iyer, "Cross layer design optimizations in wireless protocol stacks", Computer Communications, vol.27, no. 8, pp. 720-724, August 2003.

[8] A. G. Ruzzelli, G. Hare, R. Jurdak, "MERLIN: Cross-layer integration of MAC and routing for low duty-cycle sensor networks," Ad Hoc Networks, vol. 5, no. 12, pp. 296-313, December 2007.

[9] Ji Peng, Wu Chengdong, Zhang Yunzhou, et al. DAST: a QoS-aware Routing Protocol for Wireless Sensor Network [C]// International Conference on Embedded Software and Systems (ICESS2008), 2008: 259-264. 